\title{
RADIO-GUIDED MINIINVASIVE SURGERY OF SOLITARY PARATHYROID ADENOMA AS A CAUSE OF PRIMARY HYPERPARATHYROIDISM
}

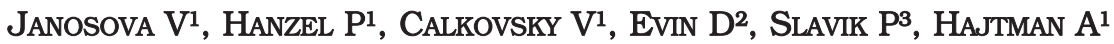 \\ ${ }^{1}$ Clinic of Otorhinolaryngology and Head and Neck Surgery, Jessenius Faculty of Medicine in \\ Martin, Comenius University in Bratislava, and Martin University Hospital, Martin, Slovakia \\ ${ }^{2}$ Clinic of Nuclear Medicine, Jessenius Faculty of Medicine in Martin, Comenius University in \\ Bratislava, and Martin University Hospital, Martin, Slovakia \\ 3Institute of Pathological Anatomy, Jessenius Faculty of Medicine in Martin, Comenius University \\ in Bratislava, and Martin University Hospital, Martin, Slovakia
}

\section{A b s t r a c t}

The function of parathyroid gland is affected by a wide spectrum of diseases, among them primary hyperparathyroidism is the most common. In about $85 \%$ of the patients a solitary parathyroid adenoma is the cause of the hyperparathyroidism. Surgical removal of pathologically changed gland is a treatment of choice, usually performed by miniinvasive radio-guided parathyroidectomy.

In this article the authors review current knowledge, research state, and present some cases of this operation at the Clinic of Otorhinolaryngology and Head and Neck Surgery, Jessenius Faculty of Medicine, Comenius University and University Hospital Martin, Martin, Slovakia.

Key words: adenoma; hyperparathyroidism; miniinvasive radio-guided parathyroidectomy

\section{INTRODUCTION}

Parathyroid gland diseases are associated with malfunction of the parathyroid gland. They include disorders causing hyperparathyroidism and hypoparathyroidism. Hypoparathyroidism occurs as a rare endocrine disorder in which parathyroid hormone (PTH) production is abnormally low or absent, but most commonly is it a result of damage or removal of parathyroid glands at the time of parathyroid or thyroid surgery.

Hypoparathyroidism is characterized by decreased function of the parathyroid glands with underproduction of parathyroid hormone, low plasma levels of calcium, cramping and twitching of muscles, or tetany. Hyperparathyroidism is the most common disorder of parathyroid glands caused by overproduction of PTH and its high plasma levels. There are three different forms of hyperparathyroidism: primary, secondary, and tertiary hyperparathyroidism (1). Primary hyperparathyroidism (PHPT) is the most common disorder of the parathyroid glands and the third most common endocrine disorder overall (12). Typically, it is characterized by high plasma levels of parathyroid hormone, hypercalcemia, and hypophosphatemia. The main cause of PHPT is a solitary parathyroid adenoma. In about $85 \%$ of the patients, a single benign tumor is the reason of the hyperparathyroidism. The remaining parathyroid glands are usually small-sized with no secretory activity. Only in 5\% of cases more than one gland is affected by the typical adenomas. Less than $15 \%$ of the cases of PHPT are caused by parathyroid hyperplasia which affects the glands asymmetrically. Glands are of normal size and showing microscopic signs of the endocrine hyperfunction. Carcinoma of parathyroid glands is seen in less than 1\% of PHPT cases. This tumor is

Corresponding author: Assoc. prof. Vladimir Calkovsky, MD, PhD.; e-mail: calkovsky@jfmed.uniba.sk

(C) 2019 Janosova $\mathrm{V}$ et al.

This work is licensed under the Creative Commons Attribution-NonCommercial-NoDerivs 4.0 License (https://creativecommons.org/licenses/by-nc-nd/4.0/) 
uncapsulated, larger and lobulated, and it adheres to the surroundings. The most of patients are asymptomatic. Symptoms are relatively rare and they are being more common in patients with malignant hypercalcemia. The most common nonspecific symptoms are weakness and fatigue, depression, bone pain, muscle soreness (myalgias), decreased appetite, feelings of nausea and vomiting, constipation, polyuria, polydipsia, cognitive impairment, kidney stones, and osteoporosis. Surgical removal of pathological gland is the most effective treatment $(1,2,5,6,9,10)$.

Secondary hyperparathyroidism occurs in the response to physiological secretion of PTH by parathyroid glands caused by hypocalcemia. The most common causes are vitamin D deficiency as a result of the lack of sunlight, diet or malabsorption, and chronic kidney failure.

Long-term secondary hyperparathyroidism results from hyperplasia of the parathyroid glands and a loss of response to plasma calcium levels. This disorder occurs most frequently in patients with end-stage kidney disease and is an autonomous activity.

In both cases, the blood test detects high levels of parathyroid hormone and hypocalcemia $(1,6)$.

Parathyroidectomy is a treatment of choice, usually performed by miniinvasive radioguided parathyroidectomy (MIP). The advantage of MIP is to remove pathologically changed gland by means of a small midline incision, less than $2-3 \mathrm{~cm}$ in length. Before MIP, a preoperative imaging by sestamibi scintigraphy is to be performed. The success of minimally invasive parathyroidectomy can be improved with the use of an intraoperative gamma probe that facilitates the surgical exploration and provides a line of sight for the surgeon (8).

This surgical technique is useful especially in the cases, when the parathyroid adenoma is identified by the pre-operative imaging by sestamibi scintigraphy. It reduces the number of reoperations, which are technically difficult due to fibrosis and altered anatomy. Moreover, it decreases the operating time and the length of hospitalization. The small size of incision accelerates the recovery. It also helps to verify the correct excision of pathological tissue (11).

MIP is indicated in patients with a scintigraphically identified solitary adenoma and with no detectable thyroid nodules showing sestamibi uptake, no history of familiar hyperparathyroidism or multiple endocrine neoplasia syndrome. Reoperation for persistent or recurrent hyperparathyroidism and ectopic adenomas are also an indication $(3,9)$.

In the next parts of the article we demonstrate the use of MIP in patients treated at the Clinic of Otorhinolaryngology and Head and Neck Surgery, Jessenius Faculty of Medicine, Comenius University (JFMCU), and University Hospital Martin (UHM) in Martin, Slovakia.

\section{CASE REPORT}

A sixty nine-year-old woman was admitted to the Clinic with the diagnosis of a primary hyperparathyroidism and the history of occasional dysphagia. Indication for surgical treatment was given by the endocrinologist.

The patient is treated for arterial hypertension, osteoarthritis, osteoporosis, chronic kidney disease grade II on the background of vascular nephrosclerosis and hyperuricemia.

A physical examination was without any abnormalities. The dual-phase parathyroid scintigraphy by 99mTc-sestamibi (methoxy isobutyl isonitrile) and 99m Tc-pertechnetate (99mTcO4) had to be completed, before the surgical treatment started.

During the first phase sestamibi is intravenously applied. This radiopharmaceutical is absorbed in the tissues of thyroid gland and hyperfunctional parathyroid gland. In the second phase the different radiopharmaceutical 99mTc-pertechnetate is applied for comparison with being concentrated merely in the thyroid gland. This preoperative phase was coordinated by the Clinic of Nuclear Medicine JFM CU and UHM.

The distribution of these two markers is visually compared with each other and the following inspection of the digital scans of thyroid gland improves the tissue visualization of the parathyroid gland $(1,7)$. 
The scintigraphy findings are shown in Fig.1. Thyroid gland is in the anatomical location. Pertechnetate distributed throughout the thyroid gland parenchyma is homogenous, without any findings of hot or cold nodules. During the second phase, the scintigraphy shows the increased tracer distribution in the area of the right caudal parathyroid gland. The size of the parathyroid gland is about $26 \times 12 \times 18 \mathrm{~mm}$.

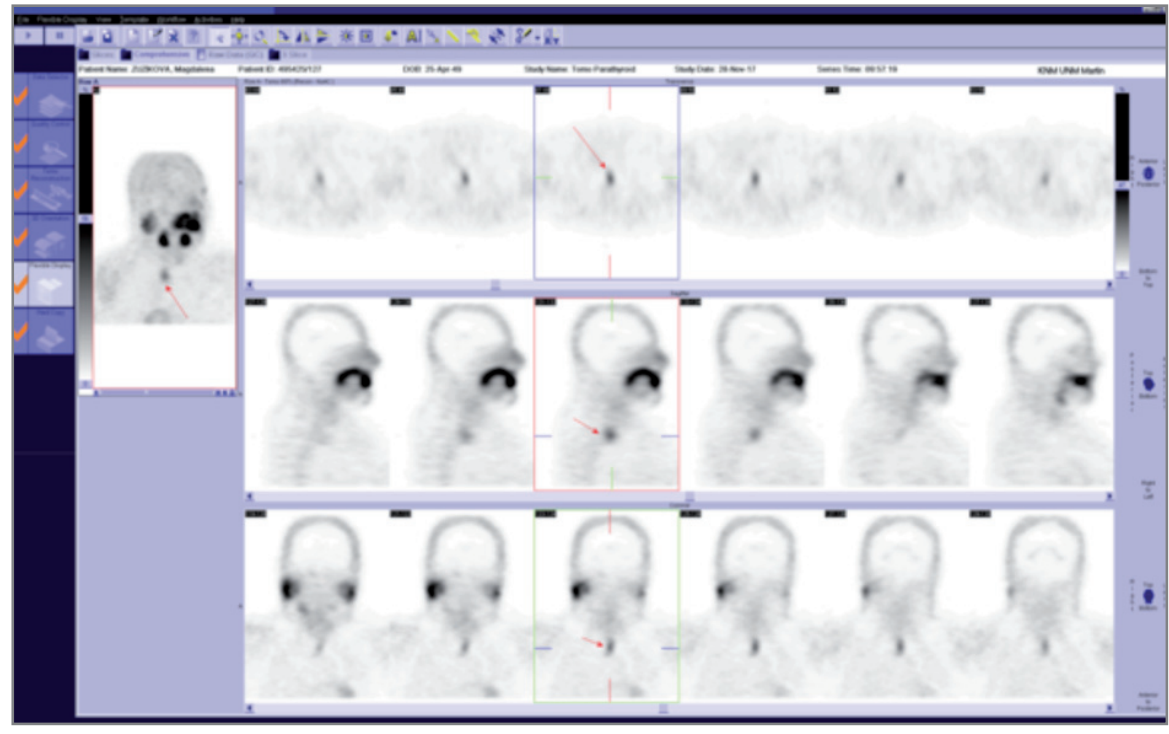

Fig. 1 Scintigraphy findings

Before the surgery the plasma levels of minerals and parathyroid hormone (PTH) were evaluated. Radiopharmaceuticals were applied in cooperation with the Clinic of Nuclear Medicine JFM CU and UHM (i.v.applied 53.37 MBq of 99mTc-MIBI) 2 hours before the surgical intervention.

A small incision through the skin was performed over the suspected adenoma in the legth of $2.5 \mathrm{~cm}$. The suspicious adenoma was identified by deep palpation (Fig. 2).

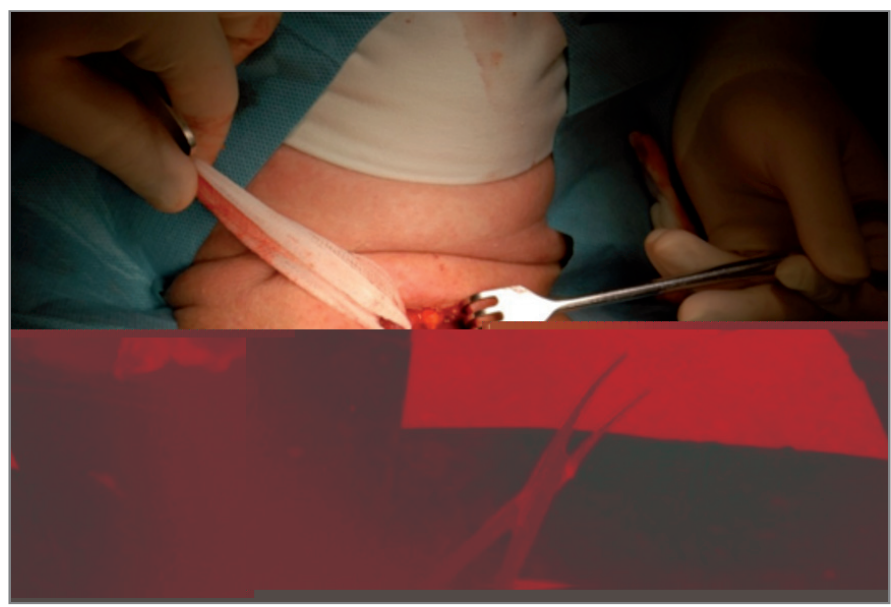

Fig. 2 The incision and palpably identified suspicious adenoma

During the surgery, the gamma probe is used (Fig. 3). It is a hand-held radiation detector with use of both auditory signals and digital counts, which guides the surgeon to dissect the radioactive target tissue (9). 
Fig. 3 Gamma probe

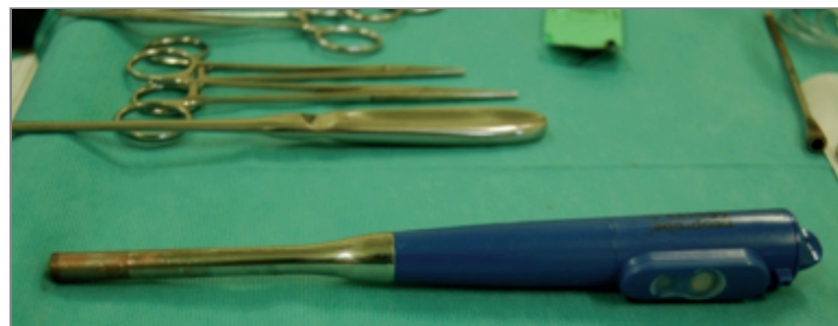

The radioactivity of the parathyroid gland, thyroid gland, and the background, usually musculus sternocleidomastoideus (m.SCM), was intraoperatively detected. The radioactivity of the parathyroid gland (Fig. 4) is more than 1.5 times higher than the thyroid (Fig. 5) and more than 2.5 - 4.5 times higher than the background (m.SCM). If the same ratio to be found, there is a great probability of the presence of the adenoma (1).

In the presented patient, the radioactivity of the adenoma was approximately 4.5 times higher than the background and 2.5 times higher than the radioactivity of thyroid gland.
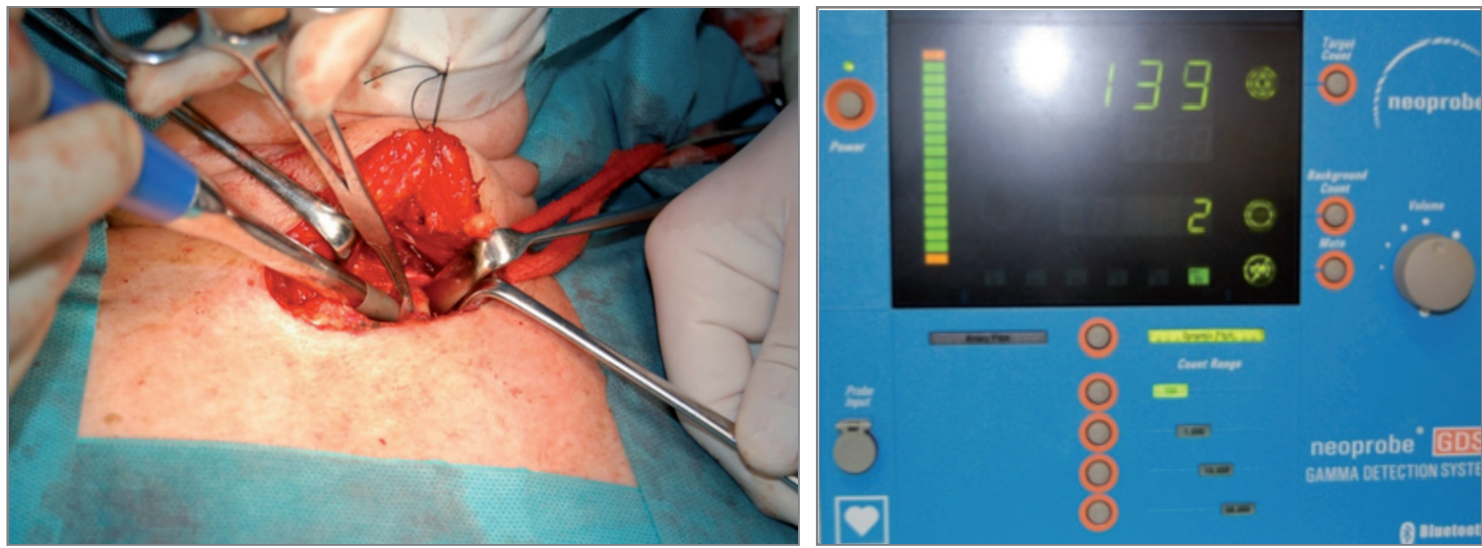

Fig. 4. Radioactivity of parathyroid adenoma
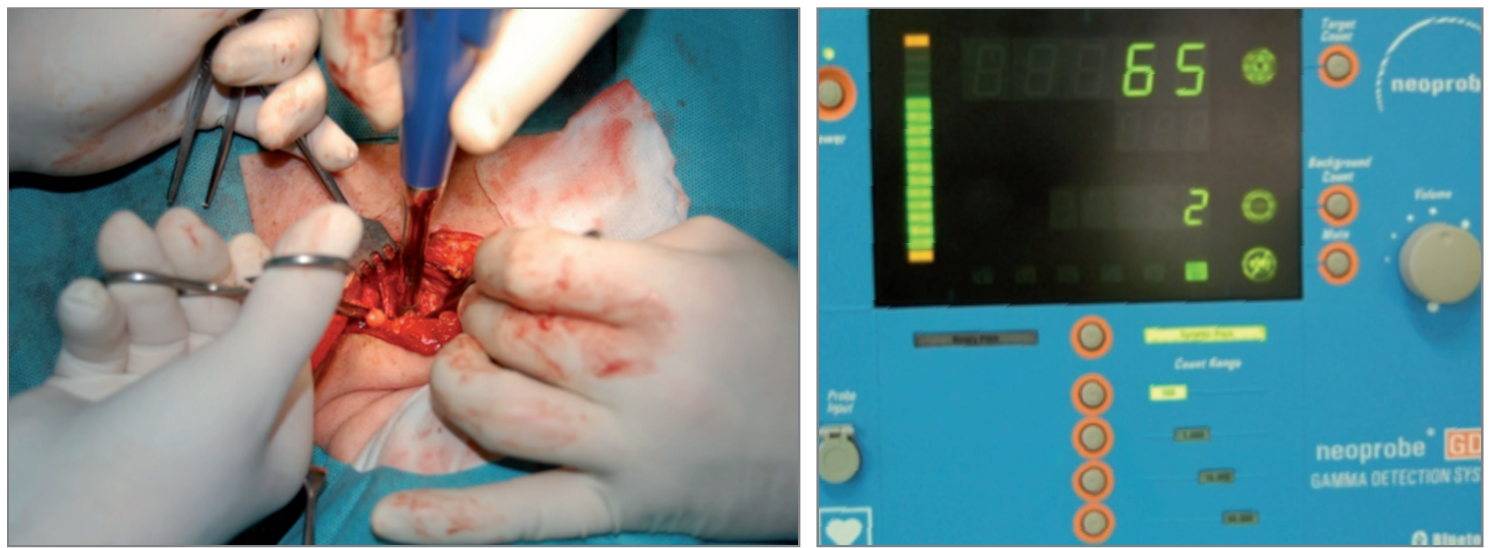

Fig. 5. Radioactivity of thyroid gland 
The size of the adenoma was approximately $40 \times 35 \times 20 \mathrm{~mm}$ and it was extirpated all (Fig. 6)

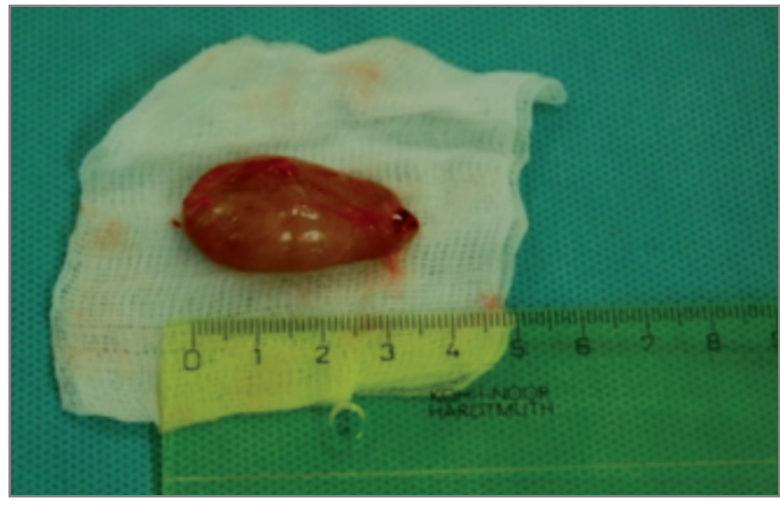

Fig. 6. Parathyroid adenoma

The histologic confirmation of the parathyroid adenoma was performed by the histopathologist in in the frozen section. The serum level of PTH should drop by half in 15-20 min after the adenoma removal. The postoperative basal value should be simultaneously within the reference range (Fig. 7).

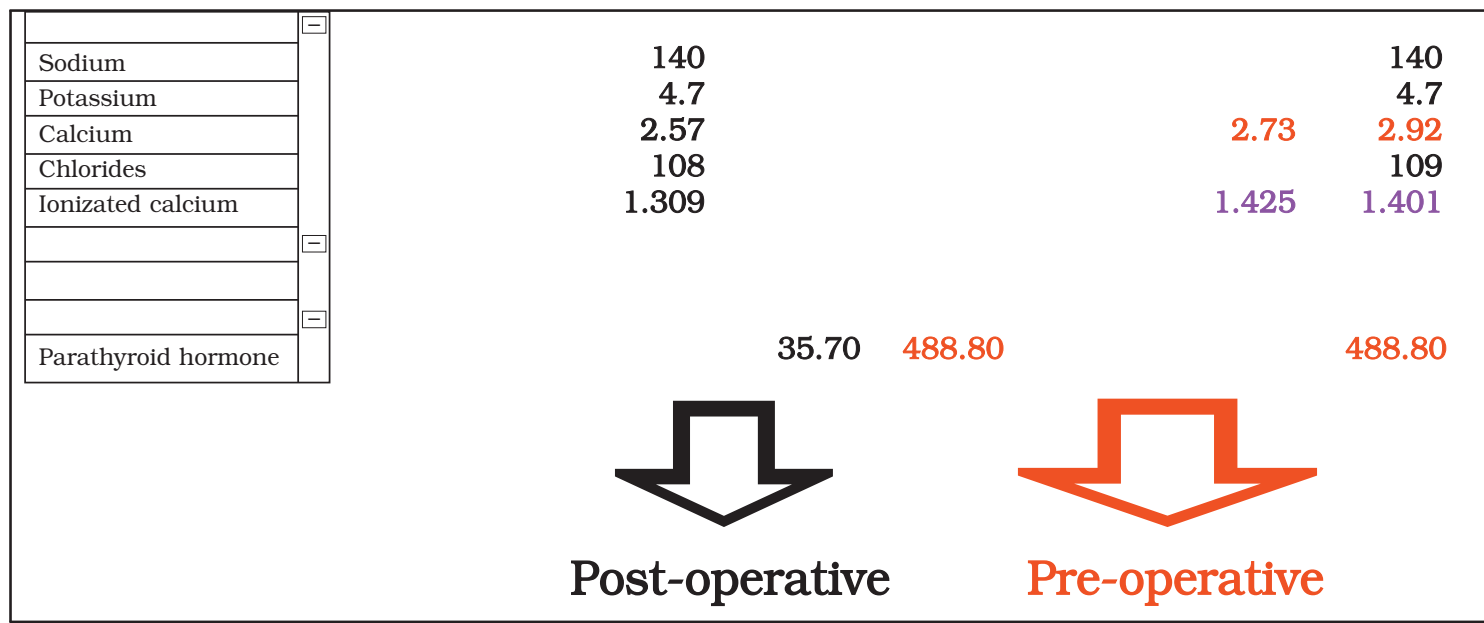

Fig. 7 Preoperative and postoperative serum level of PTH and minerals

\section{CASE SERIES - OPERATED PATIENTS}

During the period from January 2018 to May 2018, 8 patients with a diagnosis of the primary hyperparathyroidism were admitted for surgery to the Clinic of Otorhinolaryngology and Head and Neck Surgery of JFM CU and UHM.

The tracers were intravenously applied to all of them preoperatively 2 hours before the surgery. Successful extirpation of a single parathyroid adenoma was performed in 3 patients. The signals measured by the gamma probe followed recommended signals mentioned above. Parathyroid adenoma was also confirmed by the frozen section. The level of PTH postoperatively decreased to normal physiological values.

In 5 patients extirpation of the parathyroid adenoma including total thyroidectomy caused by nodular changes of the thyroid gland was indicated. In 3 of these 5 patients the 
radioactive tissue of adenoma by the gamma probe and frozen section was identified. In one patient it was not possible to identify the adenoma by the gamma probe and the tumour identification was based solely on the surgeon's experiences. Parathyroid adenoma was confirmed by frozen section. PTH plasma levels returned to physiological values in all patients. We were not able to identify adenoma neither using by the gamma probe nor frozen section, nor based on years of the surgeon's experience in one case. Postoperative parathyroid hormone elevation was persisting. The patient was consulted with the Clinic of Thoracic Surgery JFM CU and UHM. In one patient we performed hemithyroidectomy of right thyroidal lobe together with extirpation of adenoma of parathyroid gland. The gamma probe measured signals corresponded with the higher recommended ratio, but for macroscopically ambiguous trabecular appearance of tissue the presence of adenoma was uncertain. Frozen sections confirmed atypical adenoma. Postoperative PTH levels decreased.

\section{DISCUSSION AND CONCLUSION}

Miniinvasive radio-guided parathyroidectomy (MIP) is the most effective in patients who meet the inclusion criteria. For example, in the study from Italy in 321 of 344 patients MIP was successfully performed (93.3\%) (13) and MIP was also shown to be a valuable tool in the treatment of the reoperative neck (14). In contrast to bilateral exploration, MIP has been shown to be associated with significantly reduced complications (1.2\% vs. $3.1 \%)$, slightly enhanced cure rates $(99.4 \%$ vs. $97.1 \%)$, approximately $50 \%$ reduction in operating time ( 1.3 vs. 2.4 hours), and sevenfold reduction in the length of hospital stay (0.24 vs. 1.64 days) (15).

In the past, in the Clinic of Otorhinolaryngology and Head and Neck Surgery of JFM CU and UHM the patients with primary hyperparathyroidism were treated with traditional bilateral cervical neck exploration (4) and radio-guided mini-invasive surgery of solitary parathyroid adenoma was included recently as a standard surgical treatment. The cooperation with the Clinic of Nuclear Medicine is important.

MIP significantly assists to the surgeon to identify an adenoma and reduces the number of surgery failures. It is technically easy. It is more effective surgical procedure than bilateral neck exploration which success depends mainly on the surgeons experience and expertise in pathological recognition and physiological parathyroid glands. It reduces the number of reoperations in the field of fibrosis and deformed anatomy. This method is particularly helpful in the case of extirpation of adenomas (1).

\section{REFERENCES}

1) Hindié E, Ugur Ö, Fuster D. EANM parathyroid guidelines. Eur J Nucl Med Mol Imaging 2009; 36: 1201-1216.

2) Al Zahrani AA, Levine MA. Primary hyperparathyroidism. Lancet 1997; 349: 1233-1238.

3) Bozkurt MF, Uğur O, Hamaloğlu E, Sayek I, Gulec SA. Optimization of the gamma probe-guided parathyroidectomy. Am Surg 2003; 69: 720-5.

4) Calkovsky V, Hajtman A. Surgical treatment of primary parathyroidism. Bratisl Lek Listy 2008; 109(3): 121-124.

5) Carty SE, Worsey MJ, Virji MA. Concise parathyroidectomy: the impact of preoperative SPECT 99mTc sestamibi scanning and intraoperative quick parathormone assay. Surgery 1997; 122: 1107-1114.

6) Elgazzar A. The pathophysiologic basis of nuclear medicine. Berlin, Springer 2001; Chap. 7, Parathyroid gland: 141-46.

7) Greenspan BS, Dillehay G, Intenzo C. SNM Practice Guideline for Parathyroid Scintigraphy. Journal of Nuclear Medicine Technology 2012; 40: 1-8. 
8) Task Force on Primary Hyperparathyroidism. The American Association of Clinical Endocrinologists and the American Association of Endocrine Surgeons position statement on the diagnosis and management of primary hyperparathyroidism. Endocr Pract 2005; 11:49-54.

9) Mariani G, Gulec SA, Rubello D, Boni G, Puccini M, Pelizzo MR. Preoperative localization and radioguided parathyroid surgery. J Nucl Med 2003; 44:1443-58.

10) Palestro CJ, Tomas MB, Tronco GG. Radionuclide imaging of the parathyroid glands. Semin Nucl Med 2005; 35: 266-276.

11) Ugur O, Bozkurt MF, Rubello D. Nuclear medicine techniques for radio-guided surgery of hyperparathyroidism. Minerva Endocrinol 2008; 33:95-104.

12) Wermers RA, Khosla S, Atkinson EJ, Achenbach SJ, Oberg AL, Grant CS, Melton LJ. Incidence of Primary Hyperparathyroidism in Rochester, Minnesota, 1993-2001: An Update on the Changing Epidemiology of the Disease. J Bone Miner Res 2006; 21: 171-177.

13) Ikeda Y, Takayama J, Takami H. Minimally invasive radioguided parathyroidectomy for hyperparathyroidism. Annals of Nuclear Medicine 2010; 24.4: 233-240.

14) Pitt SC. Radioguided parathyroidectomy for hyperparathyroidism in the reoperative neck. Surgery 2009; 146.4: 592-599.

15) Starker LF. Minimally invasive parathyroidectomy. International Journal of Endocrinology 2011.

Received: February, 8, 2019

Accepted: April, 10, 2019 